J. Dairy Sci. 93:6052-6053

(c) American Dairy Science Association, 2010.

\title{
2010 ADSA Undergraduate Scholarship Recognition Awards
}

\section{Sophomores \\ (Bronze certificate)}

William Jones

Jacqueline VanBeek

Jacob Brandt

Sean Donegan

Zachary Mason

Kendra Murray

Erich Hodges

Emily Benedict

Hanna Borne

Amanda Daniels

Kristina Moore

Kristen Glosson

Philip Bachman

Christy Hindman

Sarah Weimer
Juniors
(Silver certificate)

Seniors

(Gold certificate)
California Polytechnic State University; San Luis Obispo

Mark Fagundes

Christina Puetz

\section{Cornell University}

Lucas Fuess

Allyson Jones-Brimmer

Jason McNamara

Ashley Sears

Alison Vosburg

Iowa State University

Emily Ullerich

\section{Louisiana State University}

Sara Menard

Michigan State University

Karmen Jackson

Allan Mergener

Shawnee Williams

\section{North Carolina State University}

Jesica Leonard

The Ohio State University

Andrew Lefeld

Jason Miley

The Pennsylvania State University

Laraya Foresman

Kolby McIntyre

University of Illinois

Emily Lyons
Andres Gomez

Brian Medeiros

Trevor Nutcher

Michelle Van Ryn

Margaret Dunn

Brett Feldpausch

Michelle Mitchell

Skylar Ryll

Chad Wall

Andrea Cook

Elizabeth Gaul

Kaleena Westaby

Hayley Hollier

Allison Vignes

Brittany Charpentier

Bergeron

Rosemary Rice

Katherine Jackson

Holly Parkins

Christina Egner

Andrea Eilenfeld

Daniel Gowanlock

Janelle Hartzell

Elizabeth Smith

Peter Yoder

Sarah Albert 
Elinor Opitz

Erin Henley

Lindsay Hetrick

Lauryn Singer

\section{University of Minnesota}

Abby Udermann

\section{Virginia Polytechnic Institute and State University}

Dana Gochenour

Dare Shepard

Brittany Thompson

Holley Weeks 